\title{
Airborne Hydrographic LiDAR Mapping - Potential of a new technique for capturing shallow water bodies
}

\author{
G. Mandlburger $^{\mathrm{a}}$, M. Pfennigbauer ${ }^{\mathrm{b}}$, F. Steinbacher ${ }^{\mathrm{c}}$ and N. Pfeifer ${ }^{\mathrm{a}}$ \\ ${ }^{a}$ Vienna University of Technology, Institute of Photogrammetry and Remote Sensing, Gusshausstr. \\ 27-29, A-1040 Vienna, Austria \\ ${ }^{\mathrm{b}}$ RIEGL Laser Measurement Systems GmbH, A-3580 Horn, Austria \\ ${ }^{\mathrm{c}}$ University of Innsbruck, Unit of Hydraulic Engineering, A-6020 Innsbruck, Austria
}

Email: gm@ipf.tuwien.ac.at

\begin{abstract}
In this paper, we present the general design of a hydrographic laser scanner (prototype instrument) manufactured by the company Riegl Laser Measurement Systems in cooperation with the University of Innsbruck, Unit of Hydraulic Engineering. The instrument utilizes a green laser source $(\lambda=532 \mathrm{~nm})$ capable of penetrating the water column. Very short laser pulses ( $1 \mathrm{~ns})$ are emitted and the backscattered signal is digitized enabling sophisticated waveform processing online during the flight and in post processing. In combination with a traditional topographic airborne laser scanner $(\lambda=1500 \mathrm{~nm})$ mounted on the same platform a complete hydrographic and topographic survey of the riparian foreland, the water surface and river bed can be carried out in a single campaign. In contrast to existing bathymetric LiDAR systems, the presented system uses only medium pulse energy but a high pulse repetition rate of up to $250 \mathrm{kHz}$ and, thus, focuses on a detailed description of shallow waters under clear water conditions.
\end{abstract}

Different fields of applications are discussed in the paper and the results of a first real-world test flight are presented. It is shown that: (i) the high pulse repetition rate enables a point density on the ground of the water body of approx. $10-20$ points $/ \mathrm{m}^{2}$, (ii) the short laser pulses together with waveform processing enable a discrimination between water and ground reflections of less than $25 \mathrm{~cm}$, (iii) the combination of a topographic and bathymetric laser scanner enable, for the first time, the acquisition of the geometry data for hydraulic modeling in a single survey, thus, providing a much more homogeneous data basis, and (iv) the high point density and the ranging accuracy of less than $10 \mathrm{~cm}$ enable a detailed and precise description of the river bed morphology, thus, providing an excellent data source for

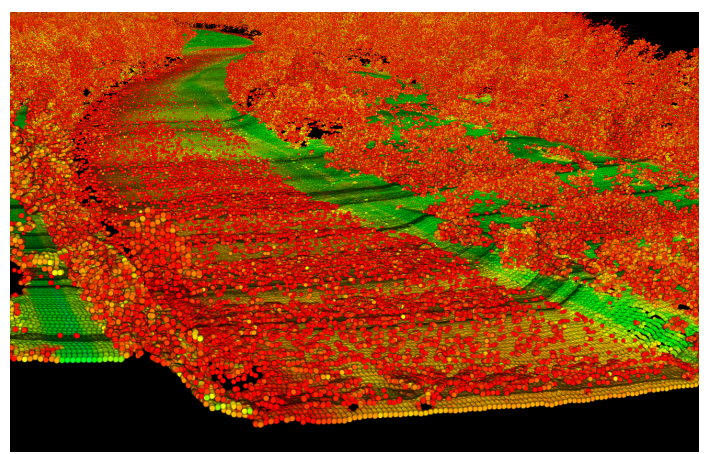

Figure 1: 3D-view of the point cloud acquired with the experimental Riegl hydrographic laser scanner VQ-820-G in July 2011

calibrating and validating sediment transport models. With the focus on capturing shallow water bodies under clear water conditions, the instrument is not designed for mapping of broader rivers (turbid water due to suspended material). However, even for these rivers the presented technique can close the gap between the river bank (captured, e.g., by topographic LiDAR) and the main channel (e.g., by echo sounding).

Keywords: Hydrography, Airborne laser bathymetry, Shallow water mapping, Full waveform analysis, Hydrology and hydraulics 


\section{INTRODUCTION}

There is a tremendous interest in mapping of shallow water bodies. A precise and detailed description of the river bed is a precondition for many applications in hydrology, hydro-morphology, hydro-biology, and hydraulics like flood simulation, sediment transport modeling and habitat mapping. Another need arises from statutory provisions like the European Water Framework Directive (WFD, EU (2000)) obligating the member states to document their fresh water resources. Furthermore, the WFD asks for repetitive survey within six years, after relevant flood events, or in case of man-made changes to the flow system.

For modeling scenes of the watercourse environment data from one sensor is typically not sufficient. This leads, next to economic disadvantages, to a number of potential problems:

- the coordinate systems, typically WGS84/UTM, have to be realized with consistent accuracy

- temporal changes in the object cause discrepancies which have to be resolved

- if the datasets are not overlapping, consistency, referring to both points above, cannot be checked.

These problems are more obvious, when similar properties are measured by the different sensors, e.g. foreland topography and river bed topography, and when these datasets are furthermore merged into one dataset, e.g. the watercourse model. While the absolute accuracy of the location of the river may be less of an issue, high relative accuracy is of crucial importance for computing correct gradients, roughness, or similar quantities. If datasets are acquired by different missions and the analysis is carried out on the joint set, the distinct datasets have to be provided with an absolute accuracy on the level of the relative accuracy. Furthermore, if data from different sensors are required for modeling of the environment, an overlap between the two data sources should be established. This allows interpreting either data source in the presence of the other source. Thus, their relative quality can be evaluated and discrepancies can, at least, be identified (Gallant and Austin, 2009). Thus, obtaining more comprehensive geo-data from one sensor is to be preferred. Requirements on absolute accuracy can be lower and the problem of the temporal basis between two acquisitions is effectively solved.

In the case of the watercourse, the currently used acquisition strategy, foreland by airborne laser scanning, water channel by terrestrial survey or echo sounding, has additional disadvantages as (i) terrestrial surveys are time and cost intensive, and provide poor data density and, thus, only few morphological details whereas (ii) waterborne echo sounding requires a certain water depth, thus, the littoral area cannot be captured. Furthermore, shallow water bodies are often near-natural featuring dense vegetation of the river bank and are, therefore, difficult to access. All these reasons advocate for using remote sensing for capturing both the riparian foreland and the river bed.

\section{STATE-OF-THE-ART}

Airborne Laser Bathymetry (ALB), also referred to as Airborne Laser Hydrography (ALH), is a method for measuring depths of shallow waters from the air. The first application of ALH was finding submarines and goes back to the mid 1960ies when the laser itself was invented. Following first successful tests, a series of systems have been developed till the end of the 1990ies: AOL (Airborne Oceanographic LiDAR) (U.S.A.), Larsen-500 (Canada), LADS (Australia), FLASH and HakeEye (Sweden), HALS (US Navy), BLOL (China) and, finally, SHOALS (U.S. Army Corps of Engineers, USACE).

The general concept of ALH involves the use of a pulsed laser transmitter with both green and infrared (IR) output (Guenther et al., 2000). The green wavelength is selected because it penetrates water with the least attenuation. IR light, in turn, penetrates water only very little and can, thus, be used for detection of the water surface (air/water interface). Knowing the speed of light in air and water, distances to the bottom of the water body can be measured based on the time-of-flight of the reflected laser pulses. In practice, a Nd:Yag laser $(\lambda=1064 \mathrm{~m})$ is often used from which the (collinear) green radiation is obtained by frequency doubling. Typical pulse repetition rates are $1 \mathrm{kHz}$ yielding an approximate point sampling distance on the ground of 4-5 m at typical flying heights $(200-500 \mathrm{~m})$. To ensure eye safety the generally collimated green laser beam is expanded to a diameter of several meters at the water surface. Most of the beam spreading, however, occurs in the water column. The maximum surveyable depth depends on system (i.e. hardware and software) and environmental conditions. The former include laser pulse energy, 
performance of the optical system, electronic noise figures, and the flying height. The latter are dominated by water clarity and bottom reflectivity. Of the two, turbidity is more important as it enters as a negative exponential factor. It can be described by the diffuse attenuation coefficient $K$ and is influenced by both scattering (redistributing energy) and absorption (reducing energy) due to dissolved organic materials and suspended organic and inorganic particles in the water.

The International Hydrographic Organization (IHO) has established a vertical accuracy standard for shallow water hydrography $( \pm 25 \mathrm{~cm}$, one sigma). To achieve such high demands, a detailed understanding of the system components and the physical interaction between the laser beam and the environment is required. Some exemplary points of importance are: (i) beam nadir angle: an angle of approx. $15-20^{\circ}$ has proved to be best suited for hydrographic surveys, the scan mechanism should ensure only small deviations of the laser beam incidence angle w.r.t. the water surface potentially compensating the aircraft's roll and pitch angles, (ii) water surface detection strategy, potentially considering different wavelengths (green, IR, and Raman (green-excited red)), and (iii) waveform recording (1 ns sampling) for sophisticated post-precessing of the range data.

More literature about the ALH principles can be found in (Guenther et al., 2000). To sum up, the existing systems use relatively long pulses $(7 \mathrm{~ns})$, high pulse energy $(5 \mathrm{~mJ})$, and low pulse repetition rates $(1 \mathrm{kHz})$. These systems are well designed for coastal applications but their applicability to riverine environments is limited as higher point densities and surveying of water depths less than $25 \mathrm{~cm}$ are required here.

\section{SENSOR CONCEPT}

The design of the experimental bathymetric laser scanner system (Riegl VQ-820-G) is described in Pfennigbauer et al. (2011), Pfennigbauer et al. (2010) and is summarized in the following. The scanner operates in the green wavelength spectrum $(\lambda=532 \mathrm{~nm})$ since signals in the wavelength range of $400-600 \mathrm{~nm}$ are capable of penetrating water columns. Very short and narrow pulses are emitted at high frequency (pulse duration: $1 \mathrm{~ns}$, beam divergence: $1 \mathrm{mrad}$, pulse repetition rate: $250 \mathrm{kHz}$ ). Short pulses $(1 \mathrm{~ns} \equiv 30 \mathrm{~cm})$ are necessary to separate returns from the water surface and the river bed in the shallow water environment the instrument is designed for. Narrow pulses $(1 \mathrm{mrad} \equiv 50 \mathrm{~cm}$ footprint diameter on the ground at a nominal flying altitude of $500 \mathrm{~m}$ ) together with the high net measurement rate $(110 \mathrm{kHz})$ ensure a high spatial resolution of typically $20-50$ points $/ \mathrm{m}^{2}$.

As can be seen in Figure 2 the green laser pulses are tilted about $20^{\circ}$ w.r.t. the flight direction. The deflection mechanism based on a rotating multi-facet mirror results in an elliptical arc-like scan pattern on the ground. This ensures that the incidence angle of the laser beam hitting a horizontal water surface does not deviate more than $\pm 1^{\circ}$ over the en-

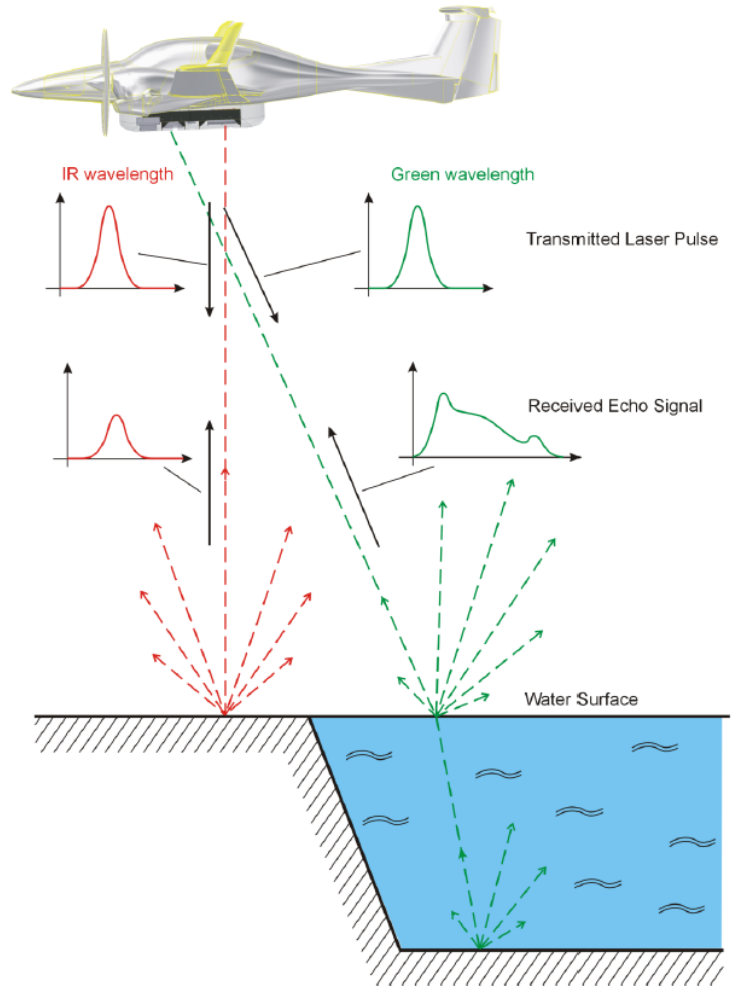

Figure 2: Combination of topographic laser scanner LMS-Q680i (near IR-wavelength) and hydrographic laser scanner VQ-820-G (green wavelength) tire scan range of $60^{\circ}$.

The instrument consists of a scan head (scan mechanism, transmitter and receiver optics, waveform processing unit) and a separate laser source housed in a 19" rack. Both components are connected via an armored glass fiber cable. This compact and lightweight design enables the installation in light aircrafts 
and helicopters. Optionally, the green laser can be coupled with a conventional topographic laser (e.g. Riegl LMS-Q680i), thus, delivering topographic data in two different wavelengths. The backscattered signal is recorded in the waveform processing unit with high temporal resolution. Online waveform processing is performed in real time during the flight mission neglecting the different propagation velocities and refraction effects in the water. The preliminary point cloud, however, enables the operator to assess the completeness of the coverage while still airborne. Refraction correction and, if necessary, more sophisticated waveform processing is performed in postprocessing in the office considering the pulse distortion due to distributed reflections, the slanted angle of incidence, refraction and the reduced propagation velocity within the water column.

The instrument is designed for measuring water depths of several meters in relatively clear water conditions. Turbidity causes distributed scattering at suspended particles attenuating the reflected signal and, thus, limiting the effective range. The maximum measurable water depth of the sensor is in the range of one Secchi depth, i.e. the depth at which the pattern of a circular black-and-white disk with a diameter of $20 \mathrm{~cm}$ is no longer visible. In contrast to existing sensors focussing on maximum depth using high pulse energy, the Riegl system is dedicated to shallow water environments and provide high point densities and range resolution utilizing only moderate pulse energy. The latter is exceptionally important w.r.t. to eye safety. The instruments' NOHD/ENOHD (i.e. eye safety for the naked/aided eye) are $100 \mathrm{~m}$ and $500 \mathrm{~m}$, respectively.

\section{Potential fields of application}

In this Section potential fields of applications of the experimental bathymetric laser scanner system are discussed.

\subsection{Hydraulics:}

Hydrodynamic-numerical simulation, also referred to as Computational Fluid Dynamics (CFD) modeling, is the state-of-the-art tool for flood prediction and prevention, disaster management, for modeling sediment transport as well as aquatic habitats. The most influencing input parameter for CFD models is the geometry provided as a Digital Terrain Model of the Watercourse (DTM-W) describing the riparian area including the river bank, the river bed, and all flow prohibiting objects like buildings or bridge piers. By now, the river bed data had to be captured independently from the flood plain topography either by single- or multi-beam echo sounding or by geodetic field survey (GPS, total station). The former poses the problem that shallow water areas (shoal, littoral zone) cannot by accessed by boat. In the latter case, cross sections at a relatively large interval of typically 20-200 m are measured requiring interpolation between two cross sections which is inaccurate as well as cost-intensive. In both cases the necessary fusion of river bed and topographic data is time-consuming and prone to errors (Gallant and Austin, 2009).

With the new hydrographic laser it is, for the first time, possible to capture all necessary geometric data for CFD modeling in a single flight campaign. That way, a homogeneous data source w.r.t. spatial resolution and height accuracy can be efficiently provided. This especially applies to small and medium rivers which are often difficult to access. Due to the required clear water conditions thorough flight planning becomes necessary. However, there exist several potential time slots distributed over the year. In the temperate climate zone these are: (i) spring after snow melt, (ii) summer keeping clear of heavy rain periods, (iii) autumn after defoliation, and (iv) winter in a moderate temperature period without snow coverage. Larger rivers, in contrast, feature higher

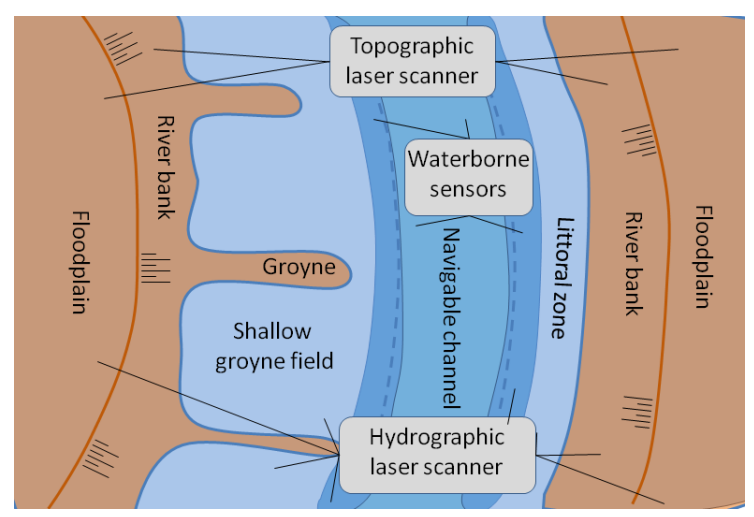

Figure 3: Potential application of hydrographic laser for surveying groyne fields turbidity due to floating sediments and/or suspended material. A complete hydrographic survey for such water bodies is not possible but, still, there is a big advantage in capturing the shallow littoral zone. 
Furthermore, the new technique bears an enormous potential for surveying shallow groyne fields (c.f. Figure 3) so that (multi-beam) echo sounding missions can be restricted to the permanently navigable channel. This would at the one hand dramatically increase the possible period of measurement as the waterborne hydrographic survey is no longer restricted to high water conditions. On the other hand, again, the fusion of topographic and hydrographic data would be facilitated and the precision increased given a certain overlap of bathymetric data from waterborne and airborne sensors. However, tests verifying this assumption are still outstanding.

\subsection{Hydro-morphology}

Due to sediment transport the morphology of the river bed is subject to constant changes. In near natural environments accumulation areas (sedimentation) periodically alternate with removal areas (degradation). Rivers regulated by impoundments, in turn, often show a deepening of the channel due to the lack of upstream sediment load. Both natural and artificial changes of the river bed morphology have an impact on the river ecosystem and, thus, influence the overall quality of the riverine environment. Therefore, repetitive surveying is a crucial task which can efficiently be achieved by airborne hydromapping. Furthermore, numerical sediment transport modeling, either loosely or tightly coupled with CFD models, gained importance in the recent past. For such models inventories of the river bed morphology at the beginning and end of the modeling period constitutes an excellent source for model calibration and validation which, up to now, had to rely on historic data with poor spatial resolution and geometric accuracy.

\subsection{Hydro-biology}

Multi-dimensional CFD models deliver estimates of spatially distributed flow velocities, water depths and bottom shear stresses for certain discharge conditions. Together with the high resolution bathymetric data this data source yields optimum qualifications for modeling aquatic habitats as it allows the differentiation of different meso-habitat types like riffle, pool, shallow water, runs, fast runs and backwaters (Hauer et al., 2009). Especially the monitoring of shallow water and backwater habitats which are exceptionally important for breeding benefit largely from the new technology as existing bathymetric data cannot compete in terms of data density and accuracy. Adolescent stages, in contrast, often prefer higher energy habitats (riffle, runs, etc.) which can also be mapped in higher detail based on airborne hydrographic data.

\subsection{River restoration}

The progressing river regulation measures of the past have contributed to an increase of the mean flow velocities. Flood events, therefore, often have a high impact on the near-shore infrastructure, residential area, and agricultural environment due to a lack of velocity detaining structures. This is only one reason why river restoration has become a topic of increasing relevance in the recent past. River restoration is a good example profiting from the combination of a topographic and hydrographic laser scanner as described in Section 2 and shown in Figure 2. Truncated ox-bow lakes and cut-off meanders are typically shallow, thus, benefit highly from the high range resolution provided by the hydrographic scanner whereas the surroundings are normally dominated by different layers of vegetation. In this case the second infrared wavelength provided by the topographic scanner helps in the vegetation analysis and ground surface extraction.

\subsection{Bankside}

One of the remaining problems when using ALS as geometric data source for CFD modeling is the embankment. In particular, if the flight mission planning favors complete areal coverage over alignment to the watercourses (e.g. countrywide ALS campaigns) this often results in sub-optimal conditions for capturing the bankside. Accurate mapping is complicated by the availability of dense river bank vegetation and by potentially inconvenient angles of incidence of the laser beam w.r.t. the bank. The compact and light weight design of both the topographic and hydrographic laser scanner allows for an installation in small and flexibly manoeuvrable airborne platforms (helicopter, light aircraft) which leads to improved conditions for deriving both the river bed and the bankside. 


\subsection{Ecology}

As mentioned before, the instrument is designed for application in clear water conditions. Turbidity, in turn, is a measure of the water quality which is demanded to be monitored on a regular basis by the European WFD. Given a sufficiently up-to-date survey of the river bed topography is available, an airborne bathymetric survey can be employed to estimate the degree of turbidity caused by sediments and suspended particles. Laser returns reflecting somewhere in the middle between the water surface and the river bed can be identified by proper waveform analysis and by comparison with the ground truth stemming from a recent flight in clear water conditions. While it is clear that not all water quality aspects demanded by the WFD can be covered by airborne hydrographic laser scanning, it will most certainly play an increasing role for topics related to ecology and water quality and not only to pure geometry.

\subsection{Monitoring}

Due to its fast and spatial survey procedure airborne laser scanning is already the state of the art technology for capturing and analyzing topographic changes. The technology of airborne hydromapping extends these possibilities to water areas. Large amounts of money are invested in river restoration, river management or water power projects. Their poor documentation and impact analysis is typically a result of missing information. Based on the new survey data and impact studies an improvement in handling water sources can potentially be achieved.

\section{First Results}

During the design phase of the instrument, experimental tests were carried out at a basin set up at the University of Innsbruck providing a typical river bed with the hydrographic laser scanner mounted on a lifting platform (Steinbacher and Pfennigbauer, 2010). The first real world test flight was conducted in February 2011 (Pfennigbauer et al., 2011). An elongated pond within a floodplain alongside the River Danube (length: $250 \mathrm{~m}$, width: $50 \mathrm{~m}$, depth: 3-4 m, Secchi depth during data capture: $\sim 4 \mathrm{~m}$ ) was surveyed with the Riegl VQ-820-G experimental hydrographic laser scanner mounted on a Eurocopter EC120 B helicopter. An arial photograph of the test site together with a color coded visualization of the resulting DTM-W, the watercourse model, an amplitude raster map, and a cross section are shown in Figure 4.

The flight took place under leaf off conditions (Figure 4a showing a summer picture of the area) during a period of very cold weather. Parts of the pond were covered with a thin layer of ice resulting in an increased number of surface returns and no ground returns in the respective area (c.f. data voids in Figure $4 \mathrm{~b}$ drawn in black). The entire pond was captured in a single flight strip featuring a very high point density of ca. 50 points $/ \mathrm{m}^{2}$. The latter is a consequence of the low flying altitude $(125 \mathrm{~m})$ and the low speed ( 25 knots). The $x-y-z$ coordinates and additional attributes amplitude and echo width were generated
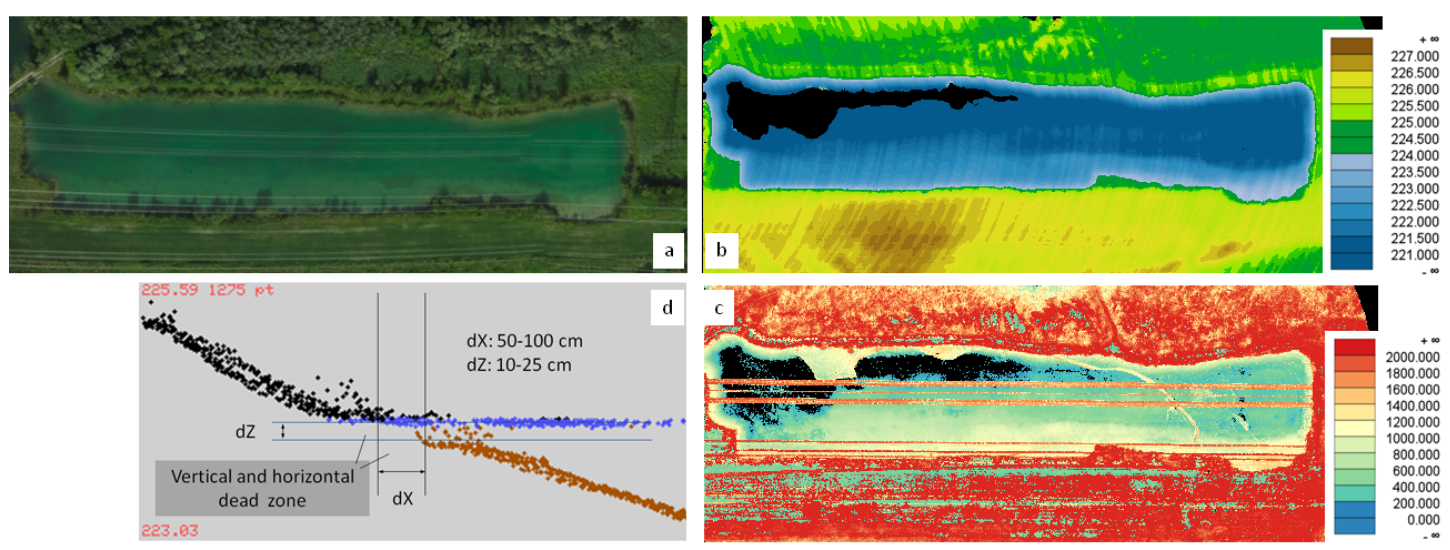

Figure 4: Hydrographic survey of a pond alongside the River Danube; (a) arial image, (b) color coded elevation map of the $0.5 \mathrm{~m}$ DTM-W grid; (c) amplitude map; (d) cross section: water surface (blue), water bottom (brown) and embankment (black) 
based on the recorded waveforms using Riegl's post processing software RiProcess. For the classification of the returns into ground, water bottom, and off-terrain (vegetation, water surface, power lines) the scientific laser scanning software OPALS (Mandlburger et al., 2009) was employed. From all ground and water bottom returns a $0.5 \mathrm{~m}$ DTM-W grid was calculated (c.f. Figure 4b). Additionally, the return amplitudes (i.e. receiver output in DN) were rasterized (maximum amplitude within $0.5 \times 0.5 \mathrm{~m}^{2}$ raster cells) and a color coded raster map was created. As expected and as can be seen in Figure $4 b+c$, the strength of the received echoes decreases with increasing water depth.

The cross section shown in Figure 4d reveals the potential of the instrument for capturing very shallow water bodies. As reported by Pfennigbauer et al. (2011), separate returns from the water surface and bottom are received as long as a minimum water depth of about $25 \mathrm{~cm}$ is exceeded. In the presented cross section, however, the vertical dead zone is even smaller. The horizontal dead zone (i.e. the distance from the shore where no bottom returns are received) generally depends on the morphology of the bank and is less than $1 \mathrm{~m}$ in the presented example.

\section{CONCLUSIONS AND OUTLOOK}

In this paper, first results of data acquisition with a hydrographic laser scanner for shallow water bodies were presented. Within a first real world test, the experimental Riegl instrument VQ-820-G successfully recorded a maximum pond depth of $4 \mathrm{~m}$ under clear water conditions. A high pulse repetition rate enables high point densities allowing to capture surface morphologies at fine scales. Due to the short pulses and dense waveform sampling the instrument is capable of measuring shallow water depths.

Potential applications of the new sensor in the fields of hydraulics, hydro-morphology, habitat modelling, river restoration, and ecology were presented and will be further investigated. More test flights together with terrestrial reference measurements have been carried out in July 2011 enabling a full cross section comparison of the airborne hydrographic data. The results will be published in the near future.

\section{ACKNOWLEDGMEnTS}

This project was supported by the Austrian Research Promotion Agency (FFG) and N. Pfeifer's contribution to this article was partly subsidized by the EU-FP7-MC-IAPP project ChangeHabitats2.

\section{REFERENCES}

EU (2000). Water Framework Directive, 2000/60/EC. Official Journal of the European Union (OJL) 327, 173.

Gallant, J. and J. Austin (2009). Stitching fine resolution dems. In Anderssen, R., Braddock, R., and Newham, L. (eds), 18th World IMACS Congress and MODSIM09 International Congress on Modelling and Simulation, July 2009: Modelling and Simulation Society of Australia and New Zealand and International Association for Mathematics and Computers in Simulation, Cairns, Australia, pp. 2486-2492.

Guenther, G. C., A. Cunningham, P. E. Laroque, and D. J. Reid (2000). Meeting the accuracy challenge in airborne lidar bathymetry. In Proceedings of the 20th EARSeL Symposium: Workshop on Lidar Remote Sensing of Land and Sea, Dresden, Germany.

Hauer, C., G. Mandlburger, and H. Habersack (2009). Hydraulically related hydro-morphological units: description based on a new conceptual mesohabitat evaluation model (mem) using lidar data as geometric input. River Research and Applications 25, 2947.

Mandlburger, G., J. Otepka, W. Karel, W. Wagner, and N. Pfeifer (2009). Orientation and Processing of Airborne Laser Scanning Data (OPALS) - Concept and First Results of a Comprehensive ALS Software. In ISPRS Workshop Laserscanning 2009, Paris, FRANCE.

Pfennigbauer, M., F. Steinbacher, A. Ullrich, and M. Aufleger (2010). A novel approach to laser-based hydrographyc data accquisition. In European Lidar Mapping Forum (ELMF) 2010, The Hague, netherlands.

Pfennigbauer, M., A. Ullrich, F. Steinbacher, and M. Aufleger (2011). High-resolution hydrographic airborne laser scanner for surveying inland waters and shallow coastal zones. In Proceedings of SPIE 8037, 8037-5, Orlando, Florida, USA.

Steinbacher, F. and M. Pfennigbauer (2010). Airborne Hydromapping - Area-wide surveying of shallow water areas. In Proceedings of the 38th ISPRS Congress, Calgary, Canada. 